\title{
Number of Stillbirths
}

National Cancer Institute

\section{Source}

National Cancer Institute. Number of Stillbirths. NCI Thesaurus. Code C139267.

A measurement of the total number of birth events at which the fetus is of at least 20 weeks gestation, or of a birth weight of at least 350 grams if the gestational age is unknown, which is born without signs of life at the time of delivery, and with an Apgar score of zero at both one and five minutes. 